\title{
LAS CRISIS FINANCIERAS Y LA EVOLUCIÓN DEL PARADIGMA EN LA PRÁCTICA DE LAS FINANZAS*
}

\author{
DOI: $10.22395 /$ seec.v19n40a2 \\ Gildardo Adolfo Vargas Aguirre* \\ Manuel Díaz Flores ${ }^{* * *}$
}

Recibido: 19 de diciembre de 2015 - Aprobado: 21 de agosto de 2016

\section{RESUMEN}

Este trabajo tiene como propósito reflexionar sobre las crisis financieras derivadas de la liberalización del sector financiero, y hace énfasis en elementos del paradigma que actualmente rige la práctica profesional en dicho sector. Para identificar los aspectos más importantes a discutir, se llevó a cabo una revisión bibliográfica, destacando sobre todo aquellas investigaciones históricas de las crisis financieras y algunos de los elementos teóricos utilizados para explicar este tipo de crisis. El artículo concluye, con algunos señalamientos preliminares, que los orígenes de las crisis financieras pueden ser de carácter tanto público como privado. Los autores revisados sugieren que es conveniente un cambio de paradigma en la práctica de las finanzas, y considerar la posibilidad de incorporar el uso sustentable de los recursos financieros en el sistema.

\section{PALABRAS CLAVE}

Crisis financiera, mercados financieros, paradigma, sector financiero.

\section{CLASIFICACIÓN JEL}

G01, G10

\section{CONTENIDO}

Introducción; 1. ¿Por qué continuar con la discusión de la crisis financiera?; 2. Sistema financiero: definición y componentes; 3. Crisis financiera: definición, clasificación y autores. Las crisis como inestabilidad del

\footnotetext{
- Este artículo surge a partir del estudio de los antecedentes y justificaciones del problema que aborda el trabajo de tesis doctoral titulado "Perspectivas estratégicas sobre finanzas sustentables", actualmente en proceso de elaboración por Gildardo Adolfo Vargas, la cual se publicará en 2018 y cuyo tutor es el Dr. Manuel Díaz, Universidad Autónoma de Aguascalientes, Aguascalientes, México. Este es un artículo de reflexión. Los autores agradecen el apoyo del editor y de su personal, así como los comentarios y sugerencias del revisor anónimo.

** Licenciado en Administración, Instituto Tecnológico de Celaya, Celaya, México. Maestro en Gestión Administrativa, Instituto Tecnológico de Celaya, Celaya, México. Estudiante del doctorado en Ciencias Administrativas, Universidad Autónoma de Aguascalientes, Aguascalientes, México. Avenida Universidad \#940, Ciudad Universitaria, C. P. 20131, Aguascalientes, Aguascalientes, México. Celular: +52 04546117358 16. Correo electrónico: al210051@edu.uaa.mx.

** Licenciado en Economía, Universidad Autónoma Metropolitana Unidad Azcapotzalco (UAM-A), México, D. F. México. Maestro en Administración Pública, Centro de Investigación y Docencia Económicas (CIDE), México, D. F. México. Doctor en Ciencias Políticas y Sociales con Orientación en Administración Pública, Universidad Nacional Autónoma de México (UNAM), México, D. F., México. Profesor-investigador, Universidad Autónoma de Aguascalientes, Aguascalientes, México. Avenida Universidad \#940, Ciudad Universitaria, C. P. 20131, Aguascalientes, Aguascalientes, México. Teléfono: +52014499108460. Correo electrónico: mdiaz@correo. uaa.mx
} 
sistema; 4. La crisis financiera y los sectores público y privado; 5. Principios y paradigmas en finanzas; 6. Desarrollo sustentable en el sistema financiero; 7. Conclusiones; Bibliografía

\title{
FINANCIAL CRISIS AND THE EVOLUTION OF THE FINANCIAL PARADIGM ABSTRACT
}

The objective of this study is to reflect upon financial crisis resulting from the liberalization of the financial sector, and emphasizes on the elements of the paradigm that currently applies to the professional practices of this sector. With the end of identifying the most important aspects to be discussed, a bibliographical review was carried out, highlighting those historic researches done on previous financial crisis and some theoretical elements used to explain this type of crisis. The article concludes that some preliminary remarks about the diverse origins of the financial crisis reveal that they can both come from the private or public sectors. The revised authors suggest that a change of paradigm is convenient in the financial practice and consider the possibility of incorporating the sustainable use of the financial resources of the system.

\section{KEY WORDS}

Financial crisis, financial markets, paradigm, financial sector

\section{JEL CLASSIFICATION}

G01, G10

\section{CONTENT}

Introduction; 1. Why continue with the financial crisis discussion?; 2. Financial system: Definition and components; 3. Financial crisis; definition, classification and authors. The crisis as system instability; 4. The financial crisis and the private and public sectors; 5 . Principles and paradigms in finance; 6 . Sustainable development in the financial system; 7 . Conclusions, Bibliography.

\section{AS CRISES FINANCEIRAS E A EVOLUÇÃO DO PARADIGMA NA PRÁTICA DAS FINANÇAS \\ RESUMO}

Este trabalho tem como propósito refletir sobre as crises financeiras derivadas da liberalização do setor financeiro, e faz ênfase em elementos do paradigma que atualmente rege a prática profissional em dito setor. Para identificar os aspectos mais importantes a discutir, se levou a cabo uma revisão bibliográfica, destacando sobre tudo aquelas investigações históricas das crises financeiras e alguns dos elementos teóricos utilizados para explicar este tipo de crise. $\mathrm{O}$ artigo conclui com alguns sinais preliminares sobre as diversas origens das crises financeiras, evidenciam que podem ser tanto de carácter público como privado. Os autores revisados sugerem que é conveniente uma mudança de paradigma na prática das finanças e considerar a possibilidade de incorporar o uso sustentável dos recursos financeiros no sistema.

\section{PALAVRAS CHAVE}

Crise financeira, mercados financeiros, paradigma, setor financeiro.

\section{CLASSIFICAÇÃO JEL}

\author{
G01, G10
}

\section{CONTEÚDO}

Introdução; 1. Por que continuar com a discussão da crise financeira?; 2. Sistema financeiro: definição e componentes; 3. Crise financeira: definição, classificação e autores. As crises como instabilidade do sistema; 4 . A crise financeira e os setores público e privado; 5 . Princípios e paradigmas em finanças; 6. 
Desenvolvimento sustentável no sistema financeiro; 7. Conclusões; Bibliografia

\section{INTRODUCCIÓN}

Este trabajo tiene como propósito reflexionar sobre las crisis financieras derivadas de la liberalización del sector financiero, y se hace énfasis en elementos del paradigma que actualmente rige la práctica profesional en el sector financiero; por consiguiente, pretende ser un llamado a la reflexión acerca del papel de los intermediarios financieros y la necesidad de involucrarse de una manera más activa en la solución y prevención de las crisis financieras. Para lograr el cometido propuesto, la metodología del presente escrito se compone de una revisión bibliográfica, centrada en la exploración de definiciones, clasificaciones y teorías sobre el sistema financiero, así como en las crisis financieras y la participación del sector privado en la generación y desarrollo de estas crisis.

Forman parte de la estructura de este artículo, en primer lugar, una explicación sobre la relevancia de continuar discutiendo el tema de la crisis financiera, centrándose principalmente en el hecho de que la inestabilidad del sistema financiero aún continúa vigente, lo cual constituye un campo de cultivo para posteriores desequilibrios financieros. Después, se desarrolla un apartado sobre la definición e integración del sistema financiero, recurriendo a definiciones de diferentes autores que, aunque son de uso común, pueden ser de ayuda para precisar los elementos básicos del sistema financiero.

En la tercera sección se aborda el tema de las crisis financieras, partiendo de su definición, clasificación y recuento de principales autores estudiosos del tema; para finalmente concluir con una exposición de la hipótesis de inestabilidad financiera como teoría de soporte para comprender el origen y dinámica de las crisis financieras. En la cuarta sección se identifican las formas en las que el sector privado tuvo injerencia en el origen y desarrollo de la última crisis financiera, así como el papel del Gobierno en la recuperación del sistema financiero en crisis y la relación que el sector privado actualmente mantiene con el Gobierno en este aspecto.

En la quinta sección se explica lo que es un paradigma, cómo es manejado en el campo de las finanzas y cuál es la influencia de dichos paradigmas en el actuar de los profesionales de las finanzas que pudieron haber conducido a tomar decisiones que finalmente contribuyeron al desarrollo de la presente crisis financiera.

En la sexta sección, se presenta un enfoque de sustentabilidad en las finanzas, el cual se considera relevante como alternativa para conducir las operaciones en el sistema financiero y evitar futuros desequilibrios del mismo. Por último, el trabajo 
concluye con el bosquejo del desarrollo de un posible enfoque en el cuidado del sistema financiero, haciendo una analogía con el cuidado del medio ambiente que se plantea en el concepto de desarrollo sustentable en el cual se sugiere que, para alcanzar tal desarrollo, se requiere un cambio en los paradigmas y principios que rigen la práctica profesional y académica en el sector financiero, y de esta manera sustituirlo por uno que dé mayor peso a las ganancias a largo plazo.

\section{1. ¿POR QUÉ CONTINUAR CON LA DISCUSIÓN DE LA CRISIS FINANCIERA?}

En el presente documento se explorarán tres aspectos relacionados con el origen de las crisis, los cuales se considera que deben ser atendidos. El tema de las crisis financieras continúa en boga ya que, por ejemplo, Shiller (2015) indica que la crisis financiera de 2008 está aún sin concluir y que, por el contrario, se generan nuevas burbujas, en este caso, en los títulos de deuda. Por esta razón, el tema de la crisis financiera continúa vigente, y engloba una serie de situaciones que requieren de estudio y solución, y para efectos de este escrito, serán de interés: 1) el debate entre la búsqueda de las ganancias a largo plazo, y a corto plazo; 2) la participación del sector privado en las acciones para revertir los efectos adversos de la crisis y 3) el cambio de paradigmas en el estudio y práctica de las finanzas.

Además, en los medios se han publicado otras señales a considerar: Liao (2015), del Foro Económico Mundial, indica que un factor de riesgo es el de la banca en la sombra, definida como la situación en que instituciones que no son bancos llevan a cabo actividades bancarias de manera indirecta, ya que el incremento de la regulación ha llevado a actividades riesgosas en dicho ámbito. Stewart (2015) señala la gestación de una posible crisis financiera, dado que las medidas empleadas para estimular la economía tras la crisis de 2008 solo se han concentrado en estimular el mercado monetario a través de la disminución de las tasas de interés, lo cual alimenta el desarrollo de burbujas financieras. Por su parte, Shaffer (2015) apunta a los movimientos a la baja en los mercados de commodities, la cada vez más notoria inestabilidad en el crecimiento económico de China y la posibilidad de alza en las tasas de interés por parte de la Reserva Federal de Estados Unidos de América.

El estudio de las vías para la recuperación de la crisis financiera llevan consigo una importancia que trasciende el mundo de los negocios. Shiller (2012) indica que las finanzas figuran como una disciplina al servicio del cumplimiento de los objetivos del ser humano y, por ende, han de servir a la sociedad. Sin embargo, el sistema financiero parece que no responde como tal, y prueba de ello son las crisis recurrentes, las cuales se han acentuado a partir del estallido de la Gran Recesión de 2008, tras la cual se han sucedido constantes episodios de volatilidad e inesta- 
bilidad financiera, lo que, de acuerdo con Keeley y Love (2011), desemboca en una baja actividad económica y en desempleo.

\section{SISTEMA FINANCIERO: DEFINICIÓN Y COMPONENTES}

La estructura y el funcionamiento del sistema financiero es otro elemento que puede ayudar a comprender las crisis, por lo que se ofrece una breve reseña desde la perspectiva de los autores sobre lo que es un sistema financiero y qué elementos los componen. Una primera referencia tiene que ver con el modelo y las definiciones expuestos en la guía de compilación de indicadores de solidez financiera del Fondo Monetario Internacional (2006).

Desde la perspectiva de Pampillón, de la Cuesta y Ruza (2009), Sartré (2009) y Baena, Hoyos y Ramírez (2012), el sistema financiero es un conjunto de instituciones, participantes e instrumentos financieros que permiten el flujo de los recursos con el propósito de otorgar créditos, oportunidades de inversión y alternativas para gestionar los riesgos, así como las actividades que dan soporte a lo anterior.

Los principales participantes del sistema financiero son las empresas, las cuales según el Fondo Monetario Internacional (2006) se pueden clasificar como entidades financieras, las cuales a su vez, se pueden subdividir en instituciones de crédito (principalmente los bancos); y en otras instituciones financieras, en las que se pueden mencionar, por ejemplo, las aseguradoras y las casas de bolsa, y no financieras, como los hogares, el gobierno y sus empresas paraestatales y el Banco Central. Estos participantes, de acuerdo con Mishkin (2013) y con el propio Fondo Monetario Internacional (2006), juegan dos roles principales: el de los intermediarios, cuyo papel es el de tomar fondos que unos participantes ahorran para proporcionárselos a otros que los requieren; el otro es el rol de los reguladores, quienes supervisan las actividades de sus intermediarios y de sus clientes. El Banco Central, además de ser regulador, desempeña actividades para soportar el sistema, tales como la emisión de moneda, la gestión de las reservas internacionales del país en cuestión y la concesión de préstamos a los bancos.

En este mismo sentido, el flujo de los fondos suele realizarse a través de activos o instrumentos financieros, los cuales son herramientas que permiten su movilidad. Según el tipo de instrumento, los participantes se reúnen o establecen puntos de acuerdo para negociar la venta y adquisición de un instrumento financiero en particular, y esto, de acuerdo con el Fondo Monetario Internacional (2006), es lo que se denomina mercado financiero. Los principales mercados financieros considerados por dicha institución son el de deuda a corto plazo (mercado monetario), la deuda 
a largo plazo (mercado de bonos), el de participaciones patrimoniales (mercado accionario o de capitales) y el mercado de instrumentos financieros derivados (mercado de derivados).

Además, para que el sistema financiero pueda funcionar de manera correcta, se requiere de un sistema de pagos, el cual, de acuerdo con el Fondo Monetario Internacional (2006), integra instrumentos, procedimientos bancarios y sistemas de transferencia interbancaria de fondos que garantizan la circulación del dinero.

Por último, aunque no constituyen un mercado financiero en un sentido estricto, el Fondo Monetario Internacional (2006) considera los mercados inmobiliarios como parte del sistema financiero, dado el impacto que dicho mercado tiene sobre la estabilidad (o inestabilidad) financiera.

\section{CRISIS FINANCIERA: DEFINICIÓN, CLASIFICACIÓN Y AUTORES. LAS CRISIS COMO INESTABILIDAD DEL SISTEMA}

A pesar de la continua evolución de los sistemas financieros, estos presentan períodos de inestabilidad en los que no se cumple con su función fundamental de proveer y asegurar el flujo de los fondos. Tales desórdenes generan escenarios preocupantes como el de la crisis financiera de 2008 y que, más adelante, ha sido conocida como la Gran Recesión, de acuerdo con Santillán (2015). Sin embargo, ¿Cómo entender una crisis financiera y sus dinámicas?

Mishkin (1992) define las crisis financieras como la repentina incapacidad de los intermediarios financieros para realizar la función de asignar fondos a los solicitantes. Bagehot (2007) describe las crisis financieras como un evento repentino que crea una gran demanda de efectivo inmediato y que causa, o tiende a causar, un pánico en el país donde el efectivo está economizado, y donde las deudas a pagar son muy grandes. La constante acerca de las crisis financieras, por lo tanto, es que se trata de un fenómeno en el cual las condiciones del sistema sufren un cambio y la asignación de fondos se ve limitada, lo que afecta la actividad económica, y conduce a etapas de recesión o depresión.

Reinhart y Rogoff (2009) indican que un rasgo común de las crisis financieras es el uso de la deuda como un factor que amplifica los daños de la crisis, ya que al restringirse los flujos de recursos financieros, las entidades que solicitaron préstamos incumplen con sus obligaciones, y esto, por ende, genera pérdidas para los intermediarios que, a su vez, les otorgaron dichos créditos. 
En Santillán (2015) se encuentra una clasificación de los orígenes de las crisis financieras, las cuales se centran en tres causas fundamentales: 1) las atribuidas al sector bancario; 2) las que se originan por la balanza de pagos (o crisis cambiarias) y 3) las que tienen un origen fiscal.

La crisis financiera bancaria, derivada de la volatilidad del entorno macroeconómico, engloba como situaciones detonantes, los cambios inesperados en los precios de los commodities, de exportación o de las tasas de interés. Santillán (2015) sitúa como ejemplo de estos tipos de crisis la de México, en 1982, y la de Rusia, en 1998.

Por su parte, los problemas microeconómicos del sistema de intermediación financiera pueden tener múltiples detonadores, ya sea porque estén muy regulados o reprimidos o, bien, porque se encuentren liberalizados y adquieran una gran complejidad. Respecto al primer caso, Santillán (2015) indica que la represión ocasiona que el capital no circule de manera eficiente en el sistema para que y llegue a los participantes que lo requieran. En el segundo caso, la liberalización y/o regulaciones laxas pueden albergar otro tipo de problemas, tales como una inadecuada gestión del riesgo crediticio, aumento de tasas de interés, posibilidad de una corrida bancaria (en la que una gran cantidad de participantes retiren su dinero del sistema a la vez), campo de cultivo para fraudes y el riesgo moral derivado de la existencia de seguros de depósito. Santillán (2015) sitúa como ejemplo de este tipo de crisis la de las hipotecas de alto riesgo de Estados Unidos de 2007.

Las crisis de balanza de pagos, también conocidas como crisis cambiarias, ocurren cuando la moneda de un país está sobrevaluada y se ve obligada a devaluarse de forma súbita. Larrosa $(1999$, p. 2) la define como "una situación en donde la confianza en la paridad fija establecida ha desaparecido, llevando a una huida masiva de reservas del banco central". Santillán (2015) indica que este tipo de crisis fue la que padeció Tailandia en 1997.

La crisis fiscal, de acuerdo con Santillán (2015), ocurrn cuando, a causa de políticas fiscales inadecuadas, el Gobierno no es capaz de hacer frente a las obligaciones internacionales adquiridas. Los ejemplos que pueden ilustrar este tipo de crisis son las que padecieron Portugal, Irlanda, Grecia y España, entre los años 2009 y 2011.

En la literatura existente sobre las crisis financieras, se identifican referentes que hacen un recuento histórico de estos fenómenos, tales como Kindleberger y Aliber (2012) y Reinhart y Rogoff (2009), que son considerados autores fundamentales para este tipo de estudios, así como obras históricas más recientes, entre las cuales se pueden contar a Marichal (2013) y Santillán (2015). 
Kindleberger (2012) destaca por qué describe el ciclo de las crisis, y para ello utiliza la hipótesis de inestabilidad financiera de Minsky como referente teórico para explicar las crisis financieras. Por su parte, Reinhart y Rogoff (2009) presentan una obra de carácter cuantitativo y empírico, para lo cual utilizan un recuento histórico de una base de datos de 66 países, por un rango de 800 años. Llama la atención que establecen como un factor determinante en el desarrollo de las crisis el uso de la deuda. Marichal (2013) señala el poco estudio realizado a la situación de los países desarrollados, ya que hasta antes de la crisis dot Com y la subprime, estos fenómenos eran propios de los países con economías emergentes. La obra que coordina Santillán (2015) está enfocada en mayor medida a la Gran Recesión de 2007-2012 (compuesta por la crisis de hipotecas subprime y la crisis de deuda soberana de la Eurozona), pero también hace un recuento de crisis anteriores para colocar el contexto y señalar las diferencias entre el fenómeno actual y las anteriores.

Los autores que hacen recuentos históricos sostienen sus obras en los trabajos de otros analistas, entre los que destacan Graciela Laura Kaminsky (análisis de relación entre crisis bancarias y cambiarias; indicadores para crisis cambiarias; contagio de las crisis; Crisis en los países emergentes en general; efecto de la liberalización financiera), Barry Eichengreen (historia del sistema monetario internacional; contagio de las crisis cambiarias; tasas de cambio y su relación con la fragilidad financiera y/o ataques especulativos), Michael Bordo (la globalización, su impacto en el sector financiero y su relación con las crisis; política monetaria y cambios en los precios de los activos; ciclos de negocios nacionales), Bradford de Long (Crecimiento económico y su relación con la inversión, así como el estudio de la fluctuación de los precios en el mercado accionario, evaluación de políticas económicas) y Frederic Mishkin (estudios sobre inflación, política monetaria, crisis en economías emergentes).

En la obra de Kindleberger y Aliber (2012), se observó que al abordar el tema de las crisis financieras, se recurre a la hipótesis de la inestabilidad financiera de Minsky, para explicar cómo es que se originan y evolucionan estos fenómenos. Dado que Kindleberger, al ser reconocido como referente en cuanto a la historia y desarrollo de las crisis financieras, utiliza esta teoría a modo de marco, se consideró necesario proporcionar una exposición breve sobre dicha teoría, la cual se presenta a continuación. A grandes rasgos, la hipótesis de inestabilidad financiera se desarrolla de la siguiente manera, según se lee en Kindleberger y Aliber (2012) y en Pastor (2009):

1. Desplazamiento: todo comienza con la diseminación de altas expectativas u oportunidades de negocio en un determinado mercado; ello genera que los inversionistas comiencen a adquirir títulos o unidades de dichos activos, lo cual, a su vez, provoca que los productores intensifiquen sus inversiones para 
satisfacer la demanda.

2. Recalentamiento: el optimismo continúa su aumento y acelera la demanda de activos o títulos; por lo tanto, su precio se incrementa. Estas adquisiciones se hacen con fines especulativos; es decir, para vender a un precio más alto a los que recién se interesan en dicho sector. Por otro lado, el sector crediticio, quien comparte dicho entusiasmo, continúa con la rebaja de exigencias e incrementa el otorgamiento de créditos.

3. Vacilación: en este punto, el optimismo sobre el sector, activo o título en cuestión comienza a disminuir. Por lo tanto, la demanda del activo se reduce, lo que conduce a la disminución del precio.

4. Apuro: en este punto, se comienza a notar el descenso de los precios. Los especuladores que adquirieron sus activos a través del crédito comienzan a experimentar dificultades, debido a que la falta de compradores y el descenso de los precios les impide allegarse del efectivo que necesitan para saldar sus adeudos.

5. Revulsión: el descenso de los precios se acentúa, a medida que los especuladores y productores experimentan dificultades para colocar los activos o títulos y se ven más presionados por sus compromisos crediticios. En este punto se comienzan a dar las primeras bancarrotas de productores y/o especuladores.

6. Pánico: se da a conocer alguna señal detonante, tales como un agudo descenso en los precios, la quiebra de algún banco o empresa importante, o noticia de algún desfalco. En este momento, todos los participantes del sector se agolpan a vender sus activos o títulos.

A manera de conclusión de este apartado, se consideró necesario averiguar sobre la vigencia de la hipótesis de inestabilidad financiera de Minsky en fenómenos contemporáneos, y al respecto se tienen, por ejemplo, los trabajos de Blackburn (2008) y de Girón y Chapoy (2009) quienes utilizan la teoría para explicar la crisis de 2008. Además, Krugman (2012) también la menciona para explicar lo que ocurrió, y señala su potencial utilidad en la previsión y prevención de este mismo fenómeno. Por último, vale la pena mencionar el trabajo de Delgado (2011) quien, a pesar de indicar que la hipótesis requiere actualizarse y fortalecerse en el aspecto teórico, reconoce la capacidad de esta teoría de explicar las crisis.

\section{LA CRISIS FINANCIERA Y LOS SECTORES PÚBLICO Y PRIVADO}

Como se indicó en párrafos anteriores, las crisis pueden provenir de desórdenes 
tanto macroeconómicos como microeconómicos, o bien, del resultado de acciones gubernamentales o privadas. En este trabajo, el interés está enfocado a las crisis financieras de origen bancario derivadas de problemas microeconómicos originados por sistemas financieros liberalizados, y en concreto, a las acciones de los intermediarios financieros. Estas acciones se dan en el marco de la desregulación de los mercados, lo cual, de acuerdo con Ciro (2012) y Marichal (2013), genera un entorno propicio para movilizar cantidades muy grandes de dinero y desarrollar instrumentos de inversión complejos y con un funcionamiento poco claro, así como francos actos de corrupción. Tal situación lleva a pensar, como indica Lagoarde (2015), que esta crisis financiera es también una crisis de valores.

El trasfondo de la necesidad de una regulación se soporta en la importancia de la transparencia en el sistema financiero. La transparencia, como la define Cortés (2005), se refiere a la buena opinión y fama atribuida a alguna persona y/o entidad en general, así como a la integridad con la que se procede en los negocios y al buen obrar. En el ámbito financiero, la transparencia es útil ya que, por ejemplo, Pastor (2009) señala que permite desarrollar medidas para controlar y prevenir las crisis basadas en la gestión de riesgos. No obstante, Ciro (2012) y Argandoña (2010) señalan como un pilar causante de las crisis financieras (y en particular, de la crisis más reciente, desatada en 2008) la falta de transparencia en los mercados, lo cual se reflejó en las operaciones realizadas con derivados, según Blackburn (2008), Vives (2010) y Ciro (2012); en los procesos de titularización, en los que las deudas hipotecarias se convirtieron en un instrumento financiero, tal como lo apunta Vives (2010), así como de la forma en que se reportan tales productos en los estados financieros de las instituciones que los adquieren. En Morales y otros (2010), así como en Ciro (2012) también se detecta falta de transparencia en cuanto a los procedimientos utilizados por las agencias para asignar las calificaciones. Por su parte, Pastor (2009), Salama (2010), Stiglitz (2010), Vives (2010) y Shiller (2012) agregan que los intermediarios financieros también contribuyen a la falta de transparencia al ofrecer productos financieros complejos a sus clientes, los cuales no podían percibir con precisión a qué tipo de riesgos se enfrentaban, y que. en la mayoría de las casos, no atendían a sus necesidades, sino que favorecían los intereses de enriquecimiento a corto plazo de los intermediarios financieros, así como la opacidad inherente en operaciones complejas, propias de los fondos de cobertura o hedge funds.

Con relación a lo anterior, Stiglitz (2010) menciona que en este tipo de crisis se generan dos fenómenos relacionados con la conducta de los intermediarios financieros: el problema de agencia y las externalidades. El problema de agencia, de acuerdo con Gitman y Zutter (2012), es aquel que se presenta cuando los administradores anteponen sus metas personales a las de los accionistas. Stiglitz (2010) 
lo contextualiza en el tema de las crisis financieras y añade que, debido a que la remuneración de los administradores está vinculada a los precios de mercado de las acciones de las empresas que administran, ellos tienden a hacer cualquier cosa para incrementar el precio de las acciones en el mercado lo más pronto posible, lo cual, en los casos más extremos, deriva en la práctica de actos deshonestos (alteración de estados financieros y / u ocultar información, por mencionar unos ejemplos). Por su parte, la externalidad es definida por Stiglitz (2010) como una situación en donde un intercambio de mercado impone costos o beneficios a terceros que no participan en dicha transacción. Al aplicar este concepto al entorno de la crisis, el autor hace alusión a todos aquellos sectores de la sociedad no relacionados con la industria financiera que han sido afectados por dicho fenómeno.

Tras el estallido de la crisis, las acciones para recuperar la estabilidad han provenido en mayor medida de los gobiernos y de las instituciones reguladoras: nuevas leyes y/o endurecimiento de las existentes, rescates bancarios, entre otros. Al respecto, Correa, Romo y Vidal (2010) indican que uno de los temas más importantes derivados por la crisis global es el de la regulación financiera, y la posibilidad de esta de cambiar las estructuras financieras para subsanar los huecos que han propiciado las condiciones de vulnerabilidad para que se genere la crisis. No obstante, Torrero (2008) explica que, si bien las autoridades son las que tienen una mayor participación en la solución de estos desastres, con frecuencia se ven obligadas a actuar bajo la presión de los mercados, sin información suficiente y en muy corto plazo, lo cual, con toda seguridad, influye en la efectividad de las medidas desarrolladas y aplicadas.

El papel de las autoridades en la recuperación de la crisis y los retos que enfrenta son reconocidos también en otras partes del mundo. De acuerdo con el documento elaborado por Watt y Botsch (2010), en el que reúnen las opiniones de investigadores, periodistas especializados y dirigentes de organismos públicos, tras el estallido de la crisis, la reacción más común fue un creciente debate sobre las medidas y regulaciones a tomar por parte del sector público, las cuales se orientan a las restricciones a las actividades de los bancos. En esta compilación, destacan medidas tales como la regulación a la banca y un mayor espectro de actuación del Gobierno en el sector financiero.

Sin embargo, el compromiso de restaurar el sistema financiero tras el desastre no puede ser exclusivo del sector público. Autores como Baker (2010); De Grauwe (2010); Kapoor (2010); Kuttner (2010) y Schuberth (2010) expresan que el problema no se resuelve únicamente con regular o traspasar las actividades privadas al sector público, ya que solo se transfiere la responsabilidad. Además, Torrero (2008) 
señala que la responsabilidad unilateral de reparar el daño causado va de la mano con el riesgo moral por parte de los ejecutivos, de ocultar información o, en otros términos, de no involucrarse en las tareas de rescate del sistema financiero que las autoridades emprenden.

Por último, cabe mencionar que Krugman (2012), Shiller (2012) y Marichal (2013) señalan que estas legislaciones son transitorias, y cuando se recupera la confianza, las restricciones disminuyen y el control se relaja. Adicionalmente, Bérubé (2010) y Bonzom (2010) destacan que las presiones de las instituciones privadas han sido para suavizar y disminuir el rigor de dichas regulaciones. De lo anterior, es necesario preguntarse: ¿Qué iniciativas emprenden las organizaciones para contribuir a la búsqueda de esa sustentabilidad en el sistema financiero? Existen algunas acciones que son consecuencia de la entrada en vigor de las regulaciones antes mencionadas, pero ¿qué hay de las iniciativas nacidas en el propio sector privado?

\section{PRINCIPIOS Y PARADIGMAS EN FINANZAS}

La práctica profesional y académica de una disciplina se rige por principios y paradigmas. Para Sautu (2003), un paradigma se define como la orientación general de una disciplina o, bien, como es concebido por Lagoarde (2015), como la visión del mundo basada en un conjunto de hipótesis teóricas que gobiernan las normas y reglas de la investigación científica, las cuales, al impregnar la forma de practicar y hacer investigación, conducen de manera indirecta a cometer ciertos actos que, para el caso de la disciplina de las finanzas, están vinvulados con el origen de las crisis y los fallos financieros relacionados con las limitaciones de los intermediarios financieros.

Para el caso de la práctica profesional, Fatemi y Fooladi (2013), Gendron y Smith (2013) y Lagoarde (2015) señalan que los principios de la maximización de las ganancias a corto plazo y la remuneración a los ejecutivos con opciones sobre acciones y primas ligadas a los resultados de la empresa, aunadas a la desregulación de los mercados financieros, fue campo de cultivo para prácticas corruptas y cuestionables, ya que los ejecutivos tienden a realizar cualquier tipo de acciones para incrementar las acciones, con tal de aumentar sus ingresos personales.

En el aspecto académico, Lagoarde (2015) indica que las finanzas se rigen por un paradigma positivista funcionalista, en el cual, de acuerdo con Ardalan (2008) se asume que la sociedad tiene una existencia concreta y sigue cierto orden. Adicional a lo anterior, Lagoarde (2015) menciona que la investigación en las finanzas busca identificar las regularidades y mecanismos de causalidad para unir las diferentes entidades del entorno financiero, a través de la utilización de metodologías y protocolos 
derivados de las ciencias naturales, con vistas a identificar leyes universales. Azofra (2005) y Gómez-Bezares (2005) también destacan la tendencia a probar teorías.

Respecto a lo anterior, Gendron y Smith (2013) y van Dijk (2014) señalan que la concentración en un solo paradigma limita la amplitud de puntos de vista para solucionar los problemas de la disciplina y proporciona un escenario sesgado de los fenómenos que ocurren en la vida real. Además, se ignora el hecho de que los seres humanos no siempre se apegan a la racionalidad, y que, como mencionan Azofra (2005) y Gómez-Bezares (2005), la excesiva formalización matemática y la continua probación de teorías dificultan la aplicabilidad de los resultados de la investigación en finanzas.

\section{DESARROLLO SUSTENTABLE EN EL SISTEMA FINANCIERO}

Dadas las circunstancias descritas bajo las cuales se genera la crisis, se puede presentar una analogía entre la interacción del ser humano con su entorno natural, y el ser humano y el sistema financiero. En este sentido, de la misma forma en que la explotación desmesurada de los recursos naturales ha traído consigo desequilibrios ambientales, la especulación y el excesivo apalancamiento traen consigo estos períodos de inestabilidad y volatilidad. Continuando con la analogía, de la misma manera que se acuñó el concepto de desarrollo sustentable, con el énfasis en el uso responsable de recursos naturales, a fin de garantizar el abasto para las necesidades futuras, también debe existir algún enfoque similar para el uso de los recursos financieros.

En la actualidad, al hablar de sustentabilidad en las finanzas, las corrientes estudiadas sobre inversiones socialmente responsables o consumo responsable de recursos financieros están atadas al factor ambiental y/o social del desarrollo sustentable; sin embargo, la cuestión que interesa abordar es: ¿̇son las únicas formas de decir que el consumo o inversión es sustentable? O bien ¿cuándo se podría decir que un consumo o inversión de recursos financieros contribuye a la sustentabilidad del sistema financiero como tal?

Para responder las cuestiones anteriores, se considera que una posible pista para generar dicho cambio radica en el concepto desarrollado por Soppe (2004) acerca de las finanzas sustentables (o finanzas corporativas sustentables) las cuales concibe como un enfoque de múltiples atributos sobre las finanzas corporativas que permiten relacionar e integrar la parte financiera, con la social y la ambiental de la organización. Si bien esta definición también incluye aspectos ambientales, este autor aborda la parte social de una forma más amplia, ya que, entre otras cosas, otorga a los grupos de interés un papel más significativo, sugiriendo que 
el paradigma de la propiedad sobre la firma no debería limitarse únicamente a los accionistas, sino más bien a un portafolio de grupos de interés.

Adicionalmente, Soppe (2015) lleva la propuesta de la sustentabilidad a los mercados financieros, y lo hace a través de una discusión sobre la tasa desigual de crecimiento del mercado financiero internacional y el crecimiento de la economía real. Señala como componente principal la tasa de interés, la cual está estrechamente relacionada con el crecimiento de la economía real. Para lograr la sustentabilidad de los mercados financieros, y al mismo tiempo generar un crecimiento a largo plazo, este autor propone una alineación entre las tasas de interés y la tasa de crecimiento económico (manteniendo una relación directa), así como la prevención de la acumulación de capital, con el fin último de equiparar su volumen del crecimiento con el de los bienes físicos.

\section{CONCLUSIONES}

A pesar de la variedad de orígenes y desarrollos de las crisis financieras, se pueden apreciar divisiones entre aspectos macroeconómicos y microeconómicos, así como entre aspectos de incumbencia del sector gubernamental y del sector privado. Si bien los estudios existentes señalan la importancia de la actuación del Gobierno, no solo como causante sino responsable de solucionar los desequilibrios ocasionados por la crisis, es necesario observar también la responsabilidad de los agentes privados en la generación de burbujas especulativas (para cuya explicación se recurrió a la hipótesis de inestabilidad financiera de Minsky), originadas, a su vez, por las prácticas derivadas del paradigma reinante en la práctica de las finanzas, el cual insta a la búsqueda de ganancias a corto plazo, y en el cual los agentes buscan su retribución en primer lugar.

Aunque el Gobierno y las instituciones reguladoras pueden imponer límites para corregir el camino, dichas legislaciones son de carácter transitorio. Para que la solución sea en verdad duradera, se requiere la participación activa del sector privado, el cual, a su vez, debe pensar en que esta crisis genera externalidades, es decir, afecta a individuos y organizaciones ajenos de primera mano a sus negocios. Por otra parte, en dichas externalidades pueden englobarse grupos de interés que las propias organizaciones tratan de cuidar en otros aspectos, dados los efectos indirectos que sí podrían tener dichos grupos sobre sus negocios. En este contexto, se propone la posibilidad de desarrollar y/o emplear un enfoque de cuidado sustentable de los recursos financieros; es decir, de atender las necesidades financieras presentes, sin comprometer las necesidades financieras futuras. 
Respecto al enfoque sustentable, si bien es incipiente y está principalmente orientado al aspecto del cuidado al medio ambiente, se exploró una propuesta de sustentabilidad en las finanzas y en el sistema financiero, que incluye elementos tanto privados como públicos. En la parte privada, vale la pena destacar el papel de la consideración de los grupos de interés como parte de los propietarios de la empresa, lo cual influiría en el tipo de decisiones que se tomarían en la firma. De esta forma, se tomarían en cuenta efectos hasta ahora minimizados. En suma, podría concluirse que la sustentabilidad en las finanzas radica en la "interiorización de las externalidades" (como lo sugieren Fatemi y Fooladi (2013)) a través de un cambio en la concepción de la firma como parte de un sistema y de su interdependencia con otros actores que, en efecto, impactan y son impactados por las decisiones financieras de la misma.

\section{BIBLIOGRAFÍA}

Ardalan, K. (2008). On the role of paradigms in finance. Aldershot: Ashgate Publishing, Ltd. 176p.

Argandoña, A. (2010). La dimensión ética de la crisis financiera. En: Mediterráneo Económico, Vol. 18, p. 183-198.

Azofra, V. (2005). Acerca de una nota crítica sobre la investigación actual en finanzas. En: Cuadernos de Economía y Dirección de la Empresa, No. 24, p. 121-149.

Baena, D., Hoyos, H., y Ramirez, J. H. (2012). Sistema financiero colombiano. Bogotá: Ecoe Ediciones. 271p.

Bagehot, W. (2007). Lombard street: a description of the money market. New York: Cosimo, Inc., $404 \mathrm{p}$.

Baker, D. (2010). Recipe for reform: accountable regulators and a smaller financial sector, p. 21-24. En: A. Watt y A. Botsch (Eds.). After the crisis: towards a sustainable growth model. Belgium: European Trade Union Institution. 186p.

Bérubé, G. (2010). Régulation financière - Wall Street restera indompté. En: Le Devoir. [En línea: 26/11/2015].

Blackburn, R. (2008). La crisis de las hipotecas subprime. En: New left review, No. 50, p. 53-95.

Bonzom, M. C. (2010). Secteur financier américain - Un réforme de nom seulement. En: Le Devoir. [En línea: 26/11/2015].

Ciro, T. (2012). Global Financial Crisis: Triggers, Responses and Aftermath. Great Britain: Ashgate Publishing, Ltd. 266p.

Correa, E., Romo, A. G. y Vidal, G. (2010). Capitalismo: ¿recuperación? ¿descomposición?. México, D.F.: Universidad Autónoma Metropolitana, Unidad Iztapalapa, División de Ciencias Sociales y Humanidades, Departamento de Economía, Miguel Ángel Porrúa. 197p.

Cortés, R. (2005). La transparencia en México: razón, origen y consecuencias. En: Revista de la 
Facultad de Derecho de México, No. 244, p. 11-32.

De Grauwe, P. (2010). The future of banking, p. 25-27. En: A. Watt y A. Botsch (Eds.). After the crisis: towards a sustainable growth model. Belgium: European Trade Union Institution, 186p.

Delgado, O. (2011). La hipótesis de la inestabilidad financiera y la crisis de 2007-2009. En: Economía: teoría y práctica, No. 34, p. 9-41.

Fatemi, A. M. y Fooladi, I. J. (2013). Sustainable finance: A new paradigm. En: Global Finance Journal, Vol. 24, No. 2, p. 101-113.

Fondo Monetario Internacional. (2006). Indicadores de solidez financiera: Guía de compilación. Washington, D.C.: International Monetary Fund. 328p.

Gendron, Y. y Smith, J. H. (2015). The global financial crisis: Essay on the possibility of substantive change in the discipline of finance. En: Critical Perspectives on Accounting, Vol. 30, p. 83-101.

Girón, A., y Chapoy, A. (2009). Financiarización y titulización: un momento Minsky. En: Economía UNAM, Vol. 16, No. 6, p. 44-56.

Gitman, L. J. y Zutter, C. J. (2012). Principios de administración financiera. 12ª edición. México, D.F.: Pearson Educación. 720 p.

Gómez-Bezares, F. (2005). Una nota crítica sobre la actual investigación en finanzas. En: Cuadernos de Economía y Dirección de la Empresa,No. 24, p. 105-120.

Kapoor, S. (2010). Principles for financial system reform, p. 17-20. En: A. Watt y A. Botsch (Eds.). After the crisis: towards a sustainable growth model. Belgium: European Trade Union Institution. 186p.

Keeley, B. y Love, P. (2011). De la crisis a la recuperación: Causas, desarrollo y consecuencias de la Gran Recesión. México, D.F.: OECD Publishing. 146p.

Kindleberger, C. P., y Aliber, R. Z. (2012). Manías, pánicos y cracs: Historia de las crisis financieras. Barcelona: Editorial Planeta. 512p.

Krugman, P. (2012). iAcabad ya con esta crisis!. España: Crítica. 272p.

Kuttner, R. (2010). Reforming finance, p. 32-35. En: A. Watt y A. Botsch (Eds.). After the crisis: towards a sustainable growth model. Belgium: European Trade Union Institution. 186p.

Lagoarde, T. (2015). Diversifying finance research: From financialization to sustainability. En: International Review of Financial Analysis, Vol. 39, p. 1 6.

Larrosa, J. M. C. (1999). Crisis de balanza de pagos: Un esquema de la teoría y evidencia hasta el presente. (Tesis de Grado). Bahía Blanca: Universidad Nacional del Sur. 32p.

Liao, M. (2015). QyA: Is another financial crisis on the way?. [En líneal World Economic Forum Agenda. [En línea: 26/11/2015].

Marichal, C. (2013). Nueva historia de las grandes crisis financieras: Una perspectiva global, 1873-2008. Buenos Aires: Debate. 424p. 
Mishkin, F. (1992). Anatomy of a financial crisis. En: Journal of Evolutionary Economics, Vol. 2, No. 2, p. 115-130.

Mishkin, F. (2013). The Economics of Money, Banking, and Financial Markets. 10 edition. United States of America: Prentice Hall. 752p.

Morales, R.; Arguedas, R.; González, J.; Oliver, M.; Martín, I. y de Pablo, R. (2010). Primeras perspectivas de la crisis y cuestiones conexas. Madrid: Editorial UNED. 381p.

Pampillón, F., de la Cuesta, M. M., y Ruza, C. (2009). Introducción al sistema financiero. Madrid: Editorial UNED. 267p.

Pastor, A. (2009). Burbujas especulativas y crisis cambiarias: revisión, prevención y cura. Barcelona: CREI, Universidad de Pompeu-Fabra, 24p.

Reinhart, C. M., y Rogoff, K. S. (2009). Esta vez es distinto: Ocho siglos de necedad financiera. México, D.F.: Fondo de Cultura Económica. 470p.

Salama, P. (2010). Una crisis financiera estructural. En: Íconos: Revista de Ciencias Sociales, No. 36, p. 19-28.

Santillán, R. J. (coord.). (2015). La Gran Recesión (2007-2012): Lecciones y oportunidades para México. México, D.F.: Fundación de Investigación IMEF. 445p.

Sastré, J. (2009). Sistema financiero mexicano. Santa Fe: El Cid Editor. 24p.

Sautu, R. (2003). Todo es teoría: Objetivos y métodos de investigación. Buenos Aires: Lumiere. $180 \mathrm{p}$.

Schuberth, H. (2010). Making finance serve society, p. 36-40. En: A. Watt y A. Botsch (Eds.). After the crisis: towards a sustainable growth model. Belgium: European Trade Union Institution. 186p.

Shaffer, L. (2015). Is EM turmoil the third wave of the financial crisis? Goldman thinks so. CNBC, [En línea: 26/11/2015].

Shiller, R. J. (2012). Las finanzas en una sociedad justa. Barcelona: Deusto, 448p.

Shiller, R. J. (2015). Exuberancia Irracional. $3^{a}$ edición. Barcelona: Deusto, 332p.

Soppe, A. (2004). Sustainable corporate finance. En: Journal of Business Ethics, Vol. 53, No. 1-2, p. 213-224.

Soppe, A. (2015). Sustainability and Long-Term Growth in the Financial Market System, p. 109123. En: Zsolnai, L. (Ed.). The Spiritual Dimension of Business Ethics and Sustainability Management. Switzerland: Springer International Publishing, 218p.

Stewart, H. (2015). Next financial crash is coming - and before we've fixed flaws from last one. The Guardian, [En línea: 26/11/2015].

Stiglitz, J. (2010). Caída libre: el libre mercado y el hundimiento de la economía mundial. Madrid: Taurus, 424p.

Torrero, A. (2008). La crisis financiera internacional y económica española. España: Encuentro, $63 p$. 
van Dijk, M. (2014). The Social Value of Finance. The Netherlands: Erasmus Universiteit Rotterdam, Erasmus School of Economics, 50p.

Vives, X. (2010). La crisis financiera y la regulación. En: Mediterráneo Económico, No. 18, p. 77-92.

Watt, A., y Botsch, A. (2010). After the crisis: towards a sustainable growth model. Belgium: European Trade Union Institution, 186p. 